only one cycle of standard 3 weekly chemotherapy. Five patients with an advanced-stage SCLC and an ECOG-PS of 4, otherwise unfit for any systemic anticancer therapy, were started on weekly chemotherapy with etoposide $\left(60-80 \mathrm{mg} / \mathrm{m}^{2}\right)$ and carboplatin (AUC 2). Four patients demonstrated a partial response (PR) while one demonstrated stable disease (SD) after 9 weeks of therapy. Improvement in PS was noted in all patients. Median progression-free survival (PFS) and overall survival (OS) were 137 and 164 days, respectively. Two patients died of disease progression, one died of massive pulmonary embolism, while two were alive and continuing on the same protocol.

Conclusion The weekly etoposide and platinum chemotherapy is a practical and feasible treatment option in patients who are otherwise fragile and unfit for standard-dose chemotherapy.

Keywords: small-cell lung cancer, lung cancer, poor performance status, weekly etoposide/platinum

\section{A Descriptive Study to Assess the Association of Geriatric Score with Observed Chemo Toxicity in Cancer Patients Older than 60 Years}

Jangala Sai Vihar ${ }^{1}$, Deepak Mulajker ${ }^{2}$

${ }^{1}$ Department of Internal Medicine, Armed Forces Medical College, Pune, Maharashtra, India

2Department of Oncology, Command Hospital, Pune, Maharashtra, India

Address for correspondence: Sai Vihar J., Armed Forces Medical College, Pune 411040, Maharashtra

(e-mail: jangala.1999saivihar@gmail.com).

\section{Abstract}

Introduction Cancer is the leading cause of death worldwide with elderly patients being predominantly affected. There seems to be a bias against administering chemotherapy to elderly patients with fewer elderly patients receiving chemotherapy as compared with their stagematched younger patients because of concerns about their capacity to endure treatment. To make personalized treatment decisions and to anticipate serious adverse effects, a toxicity prediction tool that can be computed at the bedside is the need of the hour. This well-validated score has not been tested in the Indian population. So, we decided to test the same score in our patients and try to correlate the score with the observed toxicity.

Objectives This study was aimed to determine geriatric functional status by means of a standardized geriatric score and to correlate geriatric score with observed chemo toxicity.

Materials and Methods Fifty consecutive elderly patients (age $>60$ years) with a diagnosis of cancer and scheduled for chemotherapy were recruited. These patients were evaluated using the geriatric assessment tool which is based on functional, nutritional, and psychological status. After that patient's pretherapy, chemo toxicity score or geriatric score was calculated using a published well-validated tool that consisted of 11 prechemotherapy variables as follows:

a) Age of patient,

b) Cancer type

c) Planned chemotherapy dose,

d) Planned number of chemotherapy drugs

e) Hemoglobin,

f) Creatinine clearance

g) Geriatric questions like -

i. How is your hearing?

ii. Number of falls in past 6 months?

iii. Can take your own medicines?

iv. Did your health limit you in walking one block during past 4 weeks?

v. How much of time has your physical health or emotional problems interfered with your social activities (like visiting with friends, relatives etc.)

The patients were then followed from the beginning to the end of six cycles of their chemotherapy regimen. Toxicities were noted after each clinical encounter by using the NCl-CTCAE, version 3.0.25.

Results General characteristics: the mean age of participants was 66 years (standard deviation $[S D]=4.6$ and range: $60-85$ years). Of them,
$60 \%$ received polychemotherapy and $82 \%$ received standard doses of chemotherapy. The mean score on activities of daily living was 66.7, comorbidity score was 2.7 , the psychological scale was 63.8 , the social-activity scale was 54.3 , and social-support scale was 64.1 . The mean pretherapy toxicity score is 7.24 according to the toxicity calculator. At least one grade 3 to 5 toxicity occurred in $30 \%$ of the patients ( $66 \%$ of grade $3,20 \%$ of grade 4 , and $13.3 \%$ of grade 5 ). The correlation between the predicted score and observed graded toxicity score by Pearson's scale $(\alpha=0.05)$ was 0.63 .

Conclusion The prediction model is easy to use, thus increasing the feasibility of incorporation in daily practice is important. It may enable oncologists to better assess the risk/benefit ratio and to adjust the treatment accordingly.

Keywords: geriatric, chemotherapy toxicity, myCARG

\section{Comparison of Various Radiotherapy Dose Fractionation Schedules in Palliation of Bone Metastasis}

Donald J. Fernandes ${ }^{1}$, Mariappan Senthiappan Athiyamaan', Sandesh Rao', Sharaschandra Shankar, ${ }^{1}$ Abhishek Krishna'

'Department of Radiation Oncology, Father Muller Medical College, Mangalore, Karnataka, India

Address for correspondence: Abhishek Krishna, Department of Radiation Oncology, Father Muller Medical College, Mangalore, Karnataka, 575002, India (e-mail: abhishekkrishna22@gmail.com).

\section{Abstract}

Introduction Bone metastasis is a common manifestation of malignancy. Bone metastases causes various morbidities and affect the quality of life. External beam radiotherapy is the mainstay of treatment of uncomplicated painful bone metastases. Different radiotherapy fractionation schedules are in practice for palliation of painful bone metastases.

Objectives This study was aimed to compare and report the outcomes of various fractionation schedules of radiation therapy (RT) in terms of pain relief and quality of life in patients with painful bone metastases.

Materials and Methods Eighty patients were randomized into four treatment arms with different RT fractionation schedules, namely, 8 Gy in 1 fraction, 20 Gy in 5 fractions, 24 Gy in 6 fractions, and 30 Gy in 10 fractions. Patients were assessed for pain by visual analog scale (VAS), performance status and quality of life before initiating the treatment, on the day of completion of treatment, and 1 week, 1 month, and 3 months of treatment completion.

Results Majority of the metastases constituted from breast followed by lung cancer. Of these, $27.5 \%$ had metastases to the thoracic vertebra, $26.25 \%$ to the lumbar vertebra, $22.5 \%$ to the pelvis, $8.75 \%$ to the sternum, $6.25 \%$ each to cervical vertebra and femur, and $1.25 \%$ each to humerus and ribs. The mean VAS score prior to start of RT was $5.31,5.21,5.54$, and 4.87 in arms A, B, C, and D, respectively. At the end of treatment, the scores were $3.0,3.29,2.77$, and 2.47 , respectively. At the end of 3 months, the scores were $1.54,0.57,0.54$, and 0.60 , respectively. The pain reduction was significant in all the four arms ( $p<0.05)$. Also, $25 \%$ of the patients' arm A had complete pain relief, whereas $45 \%$ of patients in arms B, C, and D had complete pain relief. In arm A, the performance status failed to improve at 3 months when compared with 1-week post-RT but the improvement was significant in the remaining three arms. There was improvement in the quality of life in all the arms, both in terms of function and symptoms. The mean score of symptomatic quality of life based on the EORTC BM22 module prior to start of RT was 38.14, 34.91, 28.85, and 29.17 in arms A, B, C, and D, respectively. There was a significant drop to $9.29,6.55,5.13$, and 6.11 at 1 -month posttreatment in the four arms, respectively. The outcomes in terms of functional quality of life showed a similar trend.

Conclusion This study demonstrated that pain reduction by various RT fractionation schedules were similar, and no statistically significant difference was noted. Performance status and quality of life improved in all the four treatment arms post-RT.

Keywords: radiotherapy, bone, metastatic, palliation 